Proceedings

\title{
A Novel Cross-Layering Power Control Mechanism for $\mathrm{AODV}^{+}$
}

\author{
Lesly Maygua-Marcillo and Luis Urquiza-Aguiar *(i)
}

Departamento de Electrónica, Telecomunicaciones y Redes de Información, Escuela Politécnica Nacional, 170109 Quito, Ecuador; lesly.maygua@epn.edu.ec

* Correspondence: luis.urquiza@epn.edu.ec; Tel.: +593-2-2976-300 (ext. 2213)

$+\quad$ Presented at the 6th International Electronic Conference on Sensors and Applications, 15-30 November 2019; Available online: https:/ / ecsa-6.sciforum.net/.

Published: 14 November 2019

\begin{abstract}
Wireless networks are technologies with a growing interest in the area of telecommunications, such as the case of MANETs. Despite its advantages, MANETs present several challenges in the transmission of information due to the limited bandwidth, high error rate, energy consumption restriction, and variable topologies. The transmission power can significantly influence some of the aforementioned issues. This paper proposes Density Power Control Mechanism (DPCM), which employs a cross-layering approach between AODV (Ad-hoc On-Demand Distance Vector) routing protocol and the physical layer to adapt power transmission. DPCM aims to reduce collisions, maintain or improve the performance of AODV as well as to save power in the nodes. Our results indicate that our proposal can improve the performance of the network and save power at the same time. Moreover, it is especially useful for low- and medium-density scenarios.
\end{abstract}

Keywords: AODV; crosslayering; MANET; power-adaptation

\section{Introduction}

A MANET [1] is a network where nodes are mobile and can be organized sporadically in arbitrary network topologies. A MANET allows people and devices to interconnect without any infrastructure as an independent network or to connect with some external network scenarios through a proper gateway. In a MANET, the nodes have a limited transmission range so to reach their destinations other nodes might route the packets. A MANET can use different wireless technologies such as Zigbee, Bluetooth, WiMAX, Wi-Fi [2]. Among the most notable features of MANETs are low cost, flexibility, decentralized architecture, easy deployment, and auto-configuration.

Despite MANETs being very versatile networks, they hardly achieve a good quality of service [2] since they are affected by the variable and unpredictable nature of the wireless channel and its dynamic topology. The transmission power is an important parameter because it determines if a transmission is successful or not, in which case, a power control would be necessary. If the transmission power is very high, the node would sense and interfere with a high number of neighbors, and it would cause channel saturation, contentions, and collisions. On the contrary, if the power is low, a node could detect very few neighbors or none, which would lead to a failed transmission. By controlling transmission power, we obtain advantages such as: increasing the number of successful transmissions, using the necessary battery energy, avoiding channel overloads and collisions. From the previous scenarios, it is reasonable to say that the power control in a MANET should be related to the operation of the upper layers, such as the network 
layer. Cooperation between layers would have great efficiency in the network, however, this approach has not been very much considered for the design of ad-hoc networks.

This paper proposes a mechanism to control the transmission power based on the detection of neighbors performed by AODV (Ad-hoc On-Demand Distance Vector). The mechanism is based on a cross-layering criterion to allow coordination, interaction, and exchange of information between the physical layer and network layer. The rest of this paper is organized as follows: Section II describes a background about routing protocols with an emphasis on AODV. Then, Section III reviews power control algorithms for MANETs. Afterward, in Section IV, we show our technique for power control using the cross-layering principle. Next, the performance evaluation is shown in Section V. Finally, we present some conclusions.

\section{Background}

Due to the mobility of the nodes in a MANET, the links between them can be formed and terminated very frequently. This fact demands that the network be able to self-organize and self-configure [3]. The path between source and destination can be modified suddenly, and when this happens, the network must be able to find a new route in the shortest time and all participating nodes must cooperate. To achieve such communication, a routing protocol with appropriate algorithms that consider the dynamic characteristics of the nodes is necessary [4]. Before the interest in wireless networks grew, wired networks used two main approaches for routing: Vector distance and Link status; however, these traditional algorithms are not useful in MANETs [5], since periodic updates of the network can consume a significant part of the available bandwidth, increase the contention on channels, and require each node to recharge its power supply frequently. For these drawbacks, a series of routing protocols for MANETs have been proposed, which can be classified according to how the routing information was obtained and maintained by the nodes. They can be divided into three different groups:

- Proactive protocols: they are a variation of the link status protocols. They determine their routes to all destinations at the beginning and keep the address information updated through a periodic process of exchange of packets. This allows a great availability of addressing requests, but it generates more signaling traffic, as well as an overload of the network. Each node has an individual routing table, for example, OLSR (Optimized Link State Routing) and DSDV (Destination-Sequenced Distance-Vector Routing) [6].

- $\quad$ Reactive protocols: Derived from protocols by distance-vector, they act on demand [7]. A route discovery process determines the correct route only when it is required by the source node [5]. In this way, it reduces unnecessary signaling traffic. AODV [8] and DSR (Dynamic Source Routing) [9] are part of this group.

- Hybrid protocols: Combine the basic characteristics of the preceding types of protocols into one. At the beginning of the routing, the route is determined proactively, and along with the network changes, the reactive type protocols are used. An example of this type is the TORA (Temporally Ordered Routing Algorithm) protocol [10].

This work uses the AODV protocol, defined in RFC 3561. It is a reactive protocol that considers an adaptation of the vector-distance algorithm adapted for mobile environments. It determines the route to the destination only in case a node wants to transmit a packet; in this way, signaling traffic is reduced and delivery time decreases [8]. The routes are maintained as long as is required by the source, provided there is connectivity between the nodes of the route. Each node has a routing table, one record per destination. Each record contains destination address, next hop to the destination, sequence number generated by the destination, and time of life. AODV manages two processes for its operation; first, make a route discovery, and then, work to maintain it. 
We have chosen AODV because of its great behavior, reported in other studies [11,12].

\section{Power Control in MANETs}

The transmission power determines the signal strength at the receiver, the transmission range, and the interference it creates for other receivers in the network. Therefore, transmission power control affects the sharing of the wireless medium and influences many aspects of the operation of wireless ad hoc networks [13]. Transmission power control is a key factor in several performance measures such as throughput delay and energy efficiency. For this reason, intensive research has been conducted to improve the network performance in terms of network throughput, delay, and energy efficiency with transmission power control. Nevertheless, these early works only consider the interplay between power control and a single layer. Hence, they may not necessarily lead to performance improvement when combining power algorithms across multiple layers. Some of the studies focused on power control using a single layer are [13]:

- Power control focused on the MAC layer, it focuses on sending packets at the minimum power needed such that the SINR in the receiver is only above the predefined threshold for successful transmission.

The methods studied have been: contention-based MAC Scheme, Interference-Based MAC Schemes, Scheduling-Based MAC Schemes [14].

- Power control focused on Network layer: Power control that operates at the MAC layer does not give routing protocols the opportunity of choosing optimal next-hop node. This means that the MAC approach only leads to local optimization of network performance, and hence it is necessary to add transmission power control at the network layer (or higher) to obtain the global optimization [13]. Authors considering the interaction between power control and network layer propose COMPOW, CLUSTERPOW, and LOADPOW schemes. The difference between these mechanisms with DPCM is that our mechanism depends on the number of neighbors detected.

Since transmission power control can affect several layers of the protocol stack, it is a typical cross-layering design problem in wireless ad hoc networks. Crosslayering design, in general, should be approached with some caution, keeping in mind the longer-term architectural issues. Kawada and Kumar [15] enumerate several principles that guide the design of transmission power control algorithms such as load effect, throughput and retransmission effect. In this paper, we consider the cross-layering technique in the mechanism for obtaining the interaction of two layers (physical and network).

\section{DPCM (Density Power Control Mechanism)}

Our objective is to add a power transmission control mechanism based on the detection of neighbors in the AODV protocol by using a cross-layering technique. For this, coordination in the exchange of information between physical and network layers is instrumental. This section details the processes, actions, and implementations that were necessary to achieve cross-layering transmission power control. First, we explain the design of our power control mechanism including its operation and the parameters considered for the calculations performed in the mechanism. After, we show how the routing protocol was modified

\subsection{Power Control Mechanism Definition}

To define the power control mechanism is necessary to consider several factors such as the number of neighbors, simulation area, transmission range, etc. The power control mechanism has been designed to be applied in MANETs with AODV routing protocol and networks with symmetrical distribution within a limited area. 
As already indicated in previous sections, the mechanism operates according to the number of neighbors that the AODV routing protocol has detected. The power value that the mechanism should increase or decrease according to the number of neighbors that it detects depends on many parameters such as simulation area, transmission range, minimum power, maximum power, and propagation loss model. The mechanism operates with a conditional logic based on the number of neighbors. Hence, according to this parameter, decisions are made to increase or reduce transmission power. The mechanism employs the following steps: (1) For DPCM to work, it is necessary to have access from the network layer to the physical layer; (2) define variables to store the power values, number of neighbors, and the addition power factor; (3) start the evaluation of the number of neighbors with the four possible cases.

For this work, it has been considered that the "ideal" number of neighbors for a node is four. This is, we set $l b=4$, one neighbor node at each cardinal point. This number has been chosen due to the uniform distribution of nodes in the simulation scenarios. For the upper bound, we will evaluate the performance of two values in Section 5.

Algorithm 1 shows our DPCM algorithm. First, in line two, if the number of neighboring nodes is zero, then the new power transmission $P$ is set to the maximum power $P_{\max }$. Second, in line three if the number of neighboring nodes is less than $l b$, then the mechanism will increase an "addition factor" (discussed later). If the neighbors detected are greater than or equal to $u b$ (line 9), the mechanism will decrease the current power of the node. This will be done by subtracting the "addition factor" from the current power. If it does not meet any condition, then the power value $P$ will be the current value. Finally, the transmission power is configured.

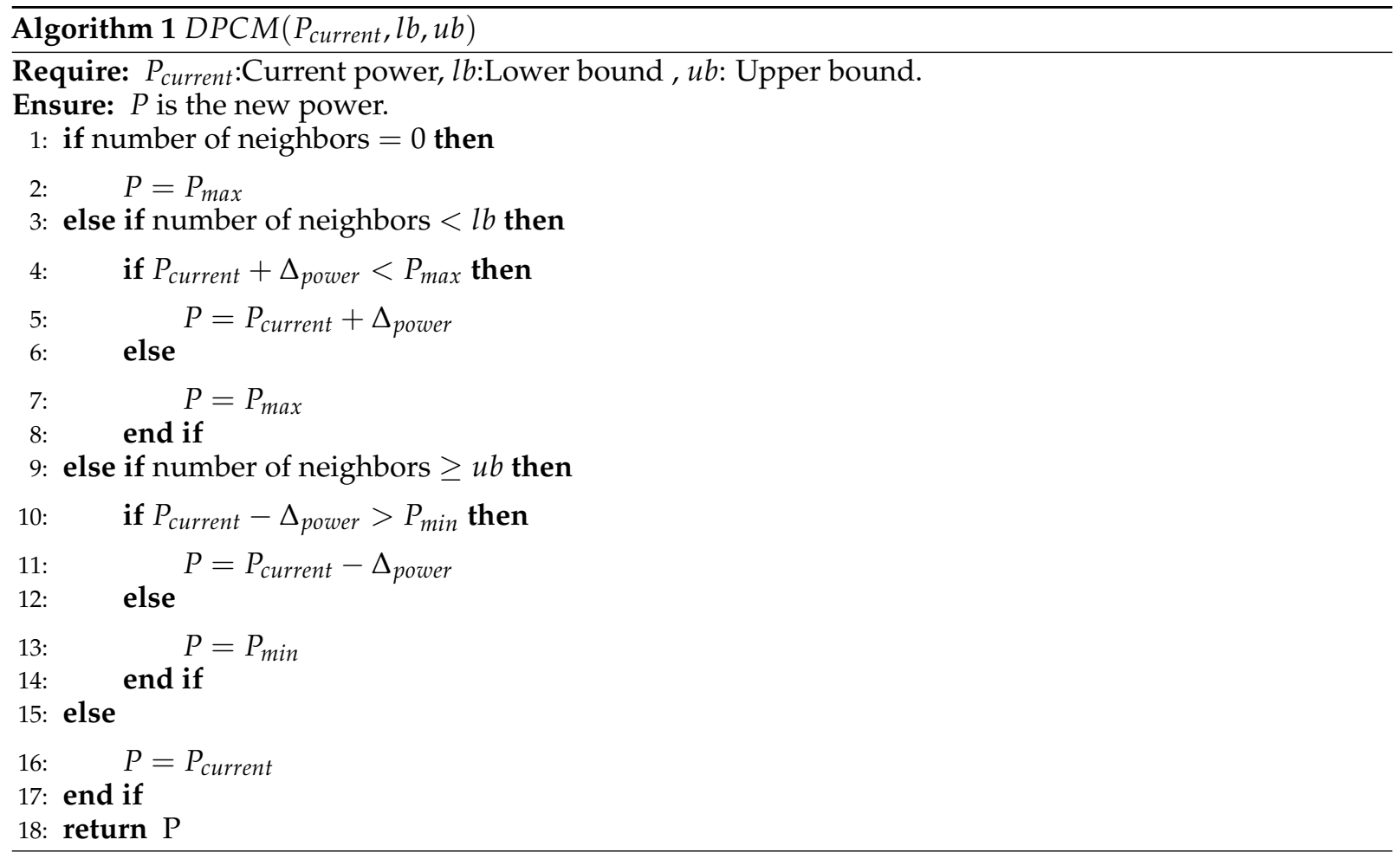

There are some parameters that the power control mechanism considers for its operation. These parameters are detailed now. 


\subsubsection{Addition Factor}

It indicates how much should be added or subtracted to the current power of a node according to Algorithm 1. This factor is calculated based on the transmission range (which depends on the channel propagation loss model), and receiver sensitivity. The actual value is obtained as a difference between the default transmission power and the transmission power needed to transmit without losses at distance $\mathrm{X}$.

By simulation, we can determine that the maximum distance to get a successful transmission using a propagation loss model based in normal log distribution is $113 \mathrm{~m}$ with the default transmission power of $16.026 \mathrm{dBm}$. Nonetheless, any more suitable propagation model can be used depending on the scenario. In this case, we calculate the loss propagation at $113 \mathrm{~m}$ as follows:

$$
L_{d=113}=L o+10 n \log (d / d o)=108.269 \mathrm{~dB}
$$

where:

$L_{o}$ : Trajectory losses at reference distance $(\mathrm{dB})$.

$d_{0}$ : Reference distance $(\mathrm{m})$.

$d$ : Distance (m).

$n$ : Exponent of path loss.

Considering the definition of reception power we have

$$
P_{i}-L_{d}=P_{R x}
$$

where:

$P_{i}$ : Default transmission power $(16.026 \mathrm{dBm})$.

$L_{d}$ : Loss propagation at distance $\mathrm{d}(\mathrm{dB})$.

$P_{R x}:$ Reception power $(\mathrm{dBm})$.

Replacing (1) in (2) we have the minimum reception power necessary to a transmission without losses.

$$
P_{\text {Rmin }}=-92.243 \mathrm{dBm}
$$

Now, it is necessary to calculate losses at a bigger distance; we consider $115 \mathrm{~m}$ using (1).

$$
L_{d=115}=\operatorname{Lo}+10 n \log (d / d o)=108.498 \mathrm{~dB}
$$

Replacing (3) and (4) in (2), we obtain a equation of the minimum power required to transmit at bigger distance without losses.

$$
P_{T x}=16.255 \mathrm{dBm}=42.218 \mathrm{mw}
$$

With (5), we can establish a difference with the initial transmission power and with that we have the addition factor.

$$
\Delta_{\text {power }}=P_{T x}-P i=2.168 \mathrm{mw}
$$

\subsubsection{Minimum Power}

It is necessary to establish a lower bound for the transmission power because there are cases in which the mechanism will decrease gradually, and in the absence of such threshold, it could be possible that power takes zero or even negative values. Thus, we include a parameter that defines the minimum power in the mechanism. The minimum power is calculated based on the density of nodes in each scenario and 
the simulation area. In this paper, the approximate value of the minimum transmission power of a node is calculated with a simple three-rule, considering the area covered by a transmission.

\subsubsection{Maximum Power}

An upper bound of the transmission power is necessary. The maximum transmission power for the IEEE 802.11a standard is $23 \mathrm{dBm}$. The ad-hoc vehicle networks book [16] indicates that the limit of transmission power in ad-hoc networks should be $20 \mathrm{dBm}$. Therefore, for the mechanism designed in this work, the upper bound is the value of $20 \mathrm{dBm}$.

\subsection{AODV Routing Protocol Modification}

The modification that we made considers three main stages: access to the physical layer from the network layer, obtaining the number of neighbors of the routing protocol, and implementing a periodic update process. For that, it is necessary to establish a link between AODV and the physical layer, which is shown in Figure 1.

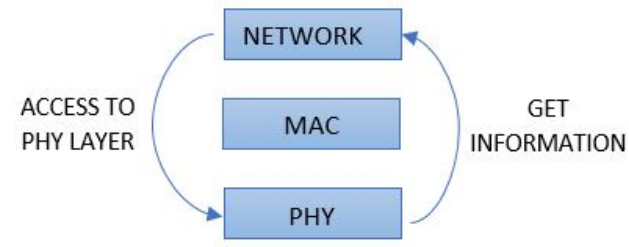

Figure 1. Link between network and physical layer.

This process includes the idea of cross-layering so that the transmission power is configured based on information that the routing protocol has sent.

Considering that the mechanism will act based on the number of neighbors that detect AODV, it is necessary to obtain all the information from neighbors to include it in the mechanism. Finally, a periodic detection process is included to get neighbor information updated constantly.

\section{Performance Evaluation}

We test the operation of our power control mechanism in the network simulator NS-3 [17]. Two scenarios will be analyzed: linear topology and a MANET with RandomWaypoint.

\subsection{Linear Topology}

We define an ad-hoc network with a group of 5 fixed nodes aligned horizontally separated by variable lengths fulfilling a total length of $370 \mathrm{~m}$ in $20 \mathrm{~s}$ of simulation. We consider a linear topology because this topology can be applied to real scenarios such as petroleum pipes and orchards [1]. Node 0 will be the server and node 4 the client, as depicted in Figure 2.

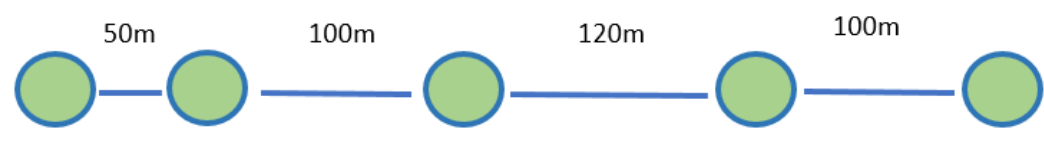

Figure 2. Linear topology.

Figure 3 shows the results obtained from the simulations with power control and without it. 


\section{WITHOUT MECHANISM \\ WITH MECHANISM}

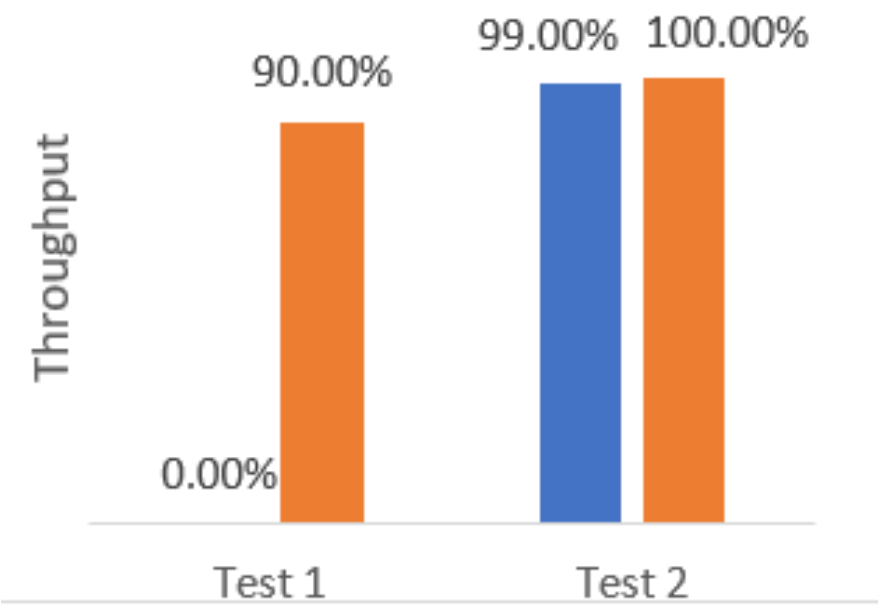

Figure 3. Analysis of throughput in a linear topology.

As can be seen, the throughput is noticeably greater when using the power control mechanism in the first test, so the functioning of the designed power control mechanism can be validated. This is because the default transmission power, which is fixed in the "without mechanism" case is not enough to create a link between nodes 2 and 3 . In the second test, the throughput obtained without using the power control mechanism achieves an almost ideal transmission; this happens because in the second test, the distance between node N2 and node N3 was reduced to $115 \mathrm{~m}$, distance at which a transmission with minimum losses is possible.

\subsection{MANET with RandomWaypoint}

Now, we consider a MANET with the RandomWaypoint mobility model. Simulations were carried out in an area of $500 \times 500 \mathrm{~m}$, considering three different densities of nodes and $\mathrm{xx}$ different speeds. For transmission, a fixed node will be the server and the remaining nodes will act as UDP clients that will send the traffic. See Figure 4. All client nodes will send 100 data packets with a size of 1024 bytes in a total simulation time of $600 \mathrm{~s}$.

In addition, for the performance evaluation, we consider two options of the mechanism. DPCM 1 refers to the power control mechanism using an upper bound $(u b)$ equal to five. On the other hand, DPCM 2 employs an upper bound equal to six. With these considerations, we analyze the performance of DPCM with respect to throughput varying the network density and speed of nodes.

\subsubsection{Throughput vs. Network Density}

This test analyzes the average throughput level that can be achieved with different density nodes (25, 36 , and 100 nodes) and with a nodes' average speed of $5 \mathrm{~km} / \mathrm{h}$. Figure 5 summarizes the results. 


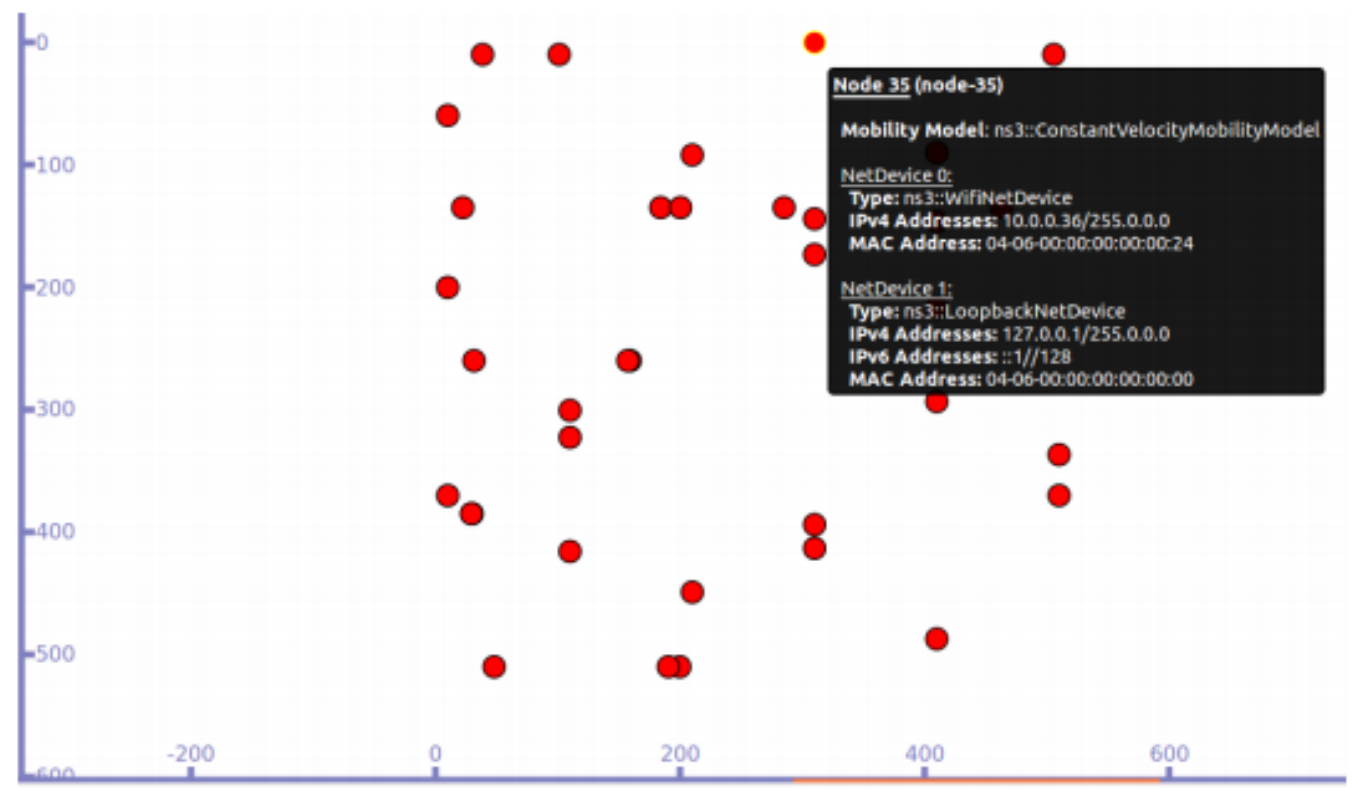

Figure 4. MANET with RandomWaypoint mobility model.

- WITHOUT MECHANISM

WITH MECHANISM

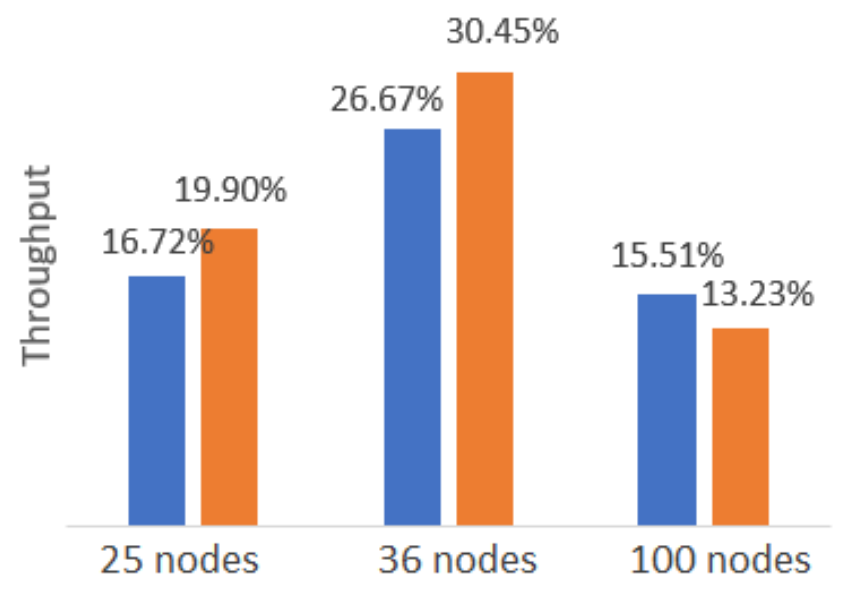

Figure 5. Analysis of throughput vs network density. DPCM 1 uses an upper bound of five. DPCM 2 uses an upper bound of six. Both DPCM configurations use a lower bound of four.

The power control mechanism will have favorable results as long as it is applied to a density not too high of nodes. When reaching the 100 nodes there is no improvement in the average throughput, this behavior is because the minimum transmission power calculated for 100 nodes is very low. This limits the successful transmissions by increasing the number of jumps (forwarding) and therefore the probability of collisions and delay.

Figure 6 shows the average transmission power for all node densities, with and without power control. 


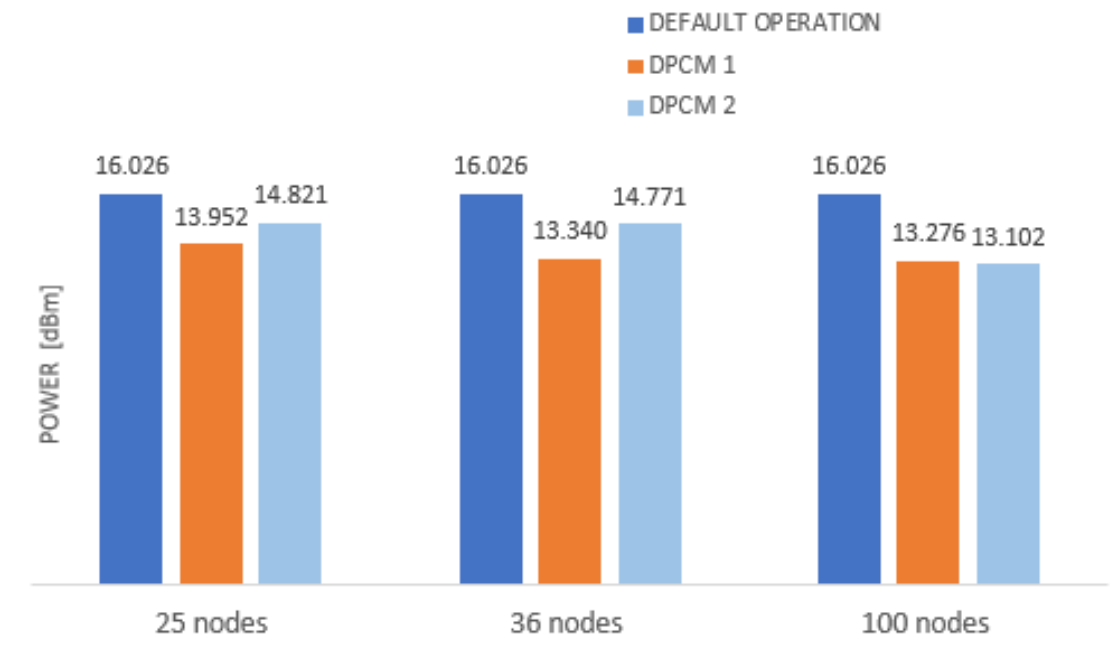

Figure 6. Analysis of average transmission power. DPCM 1 and DPCM 2 uses an upper bound of five and six respectively. Both DPCM use a lower bound of four.

The results indicate that the average transmission power was reduced when the mechanism was used. The throughput increased in two out of the three cases but, DPCM 1 using an upper bound of five nodes showed better performance. This means that the mechanism can save energy that could deteriorate the network performance.

\subsubsection{Throughput vs. Speed of Nodes}

In this case, we consider a fixed density scenario of 36 nodes and speeds of: 5,10 and $20 \mathrm{~km} / \mathrm{h}$. Figure 7 shows the average results obtained for the test with $5 \mathrm{~km} / \mathrm{h}$.

\section{- WTHOUT MECHANISM \\ WITH MECHANISM}

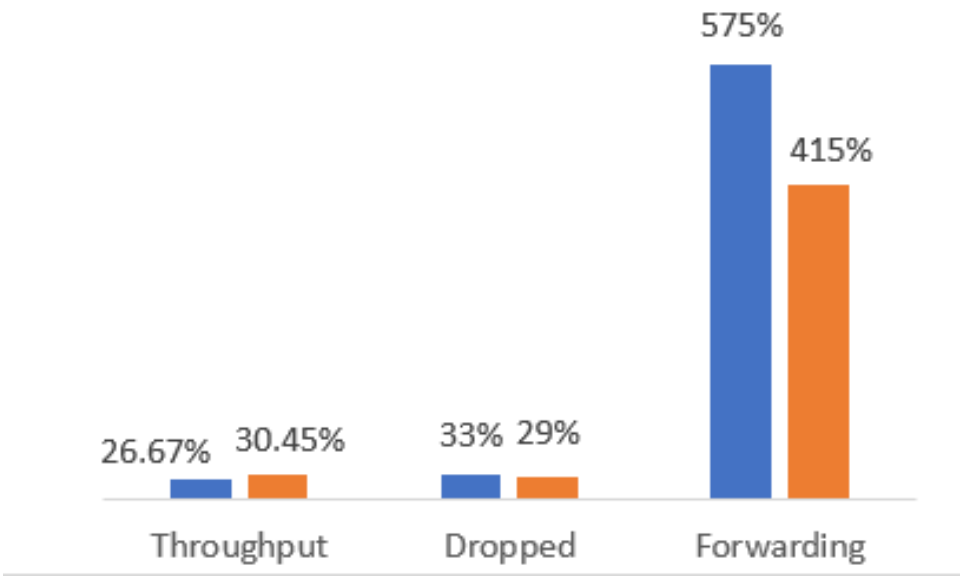

Figure 7. Throughput vs speed of nodes. DPCM 1 uses as upper bound, five. DPCM 2 uses an upper bound of six. Both DPCM configurations use a lower bound of four. 
These results analyze throughput at different speeds. The use of the power control mechanism is favorable since it allows a higher level of successful transmissions. The throughput level is higher when the speed at which the nodes move is lower, that is, at $5 \mathrm{~km} / \mathrm{h}$. For 10 and $20 \mathrm{~km} / \mathrm{h}$, it is necessary to increase the power a little more to get more lifetime of the routes.

Also, the power control mechanism allows to improve the forwarding rates for almost all cases and present a lower number of retransmissions (the number of jumps was reduced) which indicates that the mechanism designed increased the power to such a point of having longer links and therefore reducing the number of jumps. This is beneficial for the network because reduces the retransmission load, collisions, queue times and transmission time.

The evaluation of the variation of the transmission power is shown in Figure 8.

- DEFAULT OPERATION

DPCM 1

DPCM 2

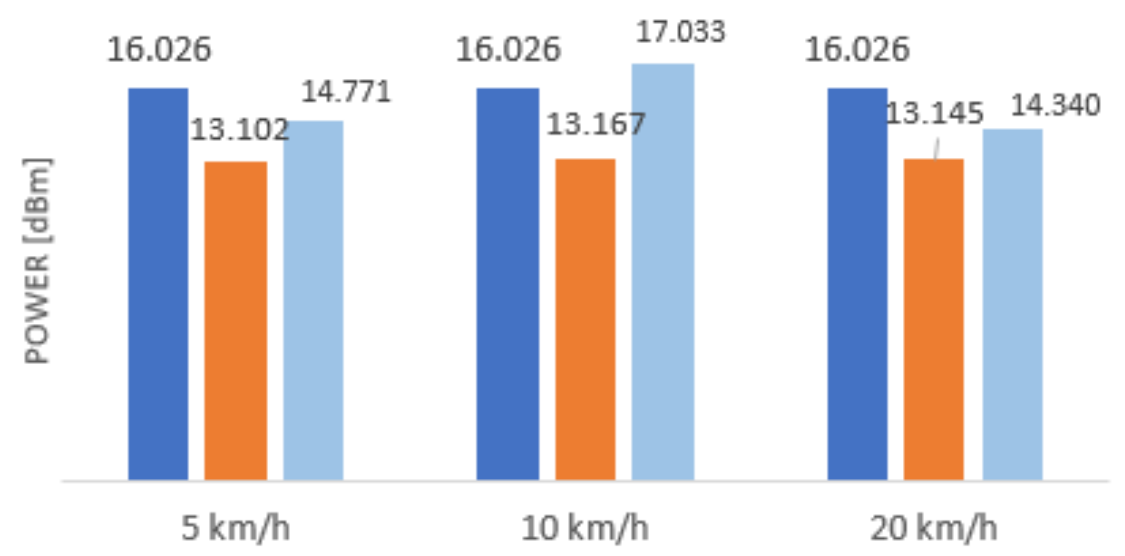

Figure 8. Analysis of average transmission power. DPCM 1 uses an upper bound of five. DPCM 2 uses an upper bound of six. Both DPCM use a lower bound of four.

The average transmission power, as in the previous tests, is lower than the power predefined by the simulator for ad-hoc networks with IEEE 802.11.

We can conclude that our power control mechanism for a MANET is beneficial in most cases. This is verified in simulations where the mechanism allows us to increase the performance. In addition, we can see that the system loses stability and the results are less favorable when the upper bound of neighbors is set above six. Moreover, the nodes' speed has a direct relation with the transmission power. As the speed of a node increases, the transmission power must increase as well to maintain a stable link to avoid fast changes in the topology of the network.

\section{Conclusions}

We have presented Density Power Control Mechanism DPCM, a straightforward mechanism to adapt the transmission power using the neighbors' information of the AODV routing protocol.

Our results show that the use of the proposed mechanism is favorable for the network in terms of efficiency and lower power consumption in most of the cases. More specifically, the designed power control mechanism operates satisfactorily at low densities of nodes. On the contrary, while the density of nodes increases, the minimum level of the transmission power is very small and therefore nodes cannot 
achieve many successful transmissions. The estimation of a good lower bound of power transmission needs further study. Another aspect that needs extensive evaluation is the number of neighbors to be set as optimal in our algorithm.

Author Contributions: Both authors contribute equally to this work. All authors have read and agreed to the published version of the manuscript

Funding: The authors gratefully acknowledge the financial support provided by the Escuela Politécnica Nacional, for the development of the project PIJ-16-01_-“Modelamiento de la Packet Error Rate (PER) incluyendo condiciones de Peak-to-Average Power Ratio (PAPR) para transmisiones Ad-Hoc".

Conflicts of Interest: The authors declare no conflict of interest.

\section{References}

1. Vyas, K.; Chaturvedi, A. Comparative Analysis of Routing Protocols in Ad-hoc Networks. In Proceedings of the 2014 International Conference on Signal Propagation and Computer Technology (ICSPCT 2014), Ajmer, India, 12-13 July 2014; pp. 692-697.

2. Urquiza, L.F. Lecture notes of Ah-hoc Networks Seminar, Quito, 2016. Available online: https:/ /www.lfurquiza. com/teaching/previouscourses/2018a/18a-adhoc-seminar (accessed on 14 November 2019).

3. Sharma, K.; Mittal, N.; Rathi, P. Comparative Analysis of Routing Protocols in Ad-hoc Networks. Int. J. Adv. Sci. Technol. 2014, 69, 1-12.

4. Mai, Y.; Bai, Y.; Wang, N. Performance Comparison and Evaluation of the Routing Protocols for MANETs Using NS3. Int. J. Electr. Eng. 2017, 5, 187-195.

5. Soujanya, B.; Sitamahalakshmi, T.; Divakar, C. Study of Routing Protocols in Mobile Ad Hoc Networks. Int. J. Eng. Sci. Technol. 2011, 3, 2622-2631.

6. Rivoirard, L.; Wahl, M.; Sondi, P.; Berbineau, M.; Gruyer, D. Performance evaluation of AODV, DSR, GRP and OLSR for VANET with real-world trajectories. In Proceedings of the 2017 15th International Conference on ITS Telecommunications (ITST), Warsaw, Poland, 29-31 May 2017; pp. 1-7.

7. Mejaele, L.; Oketch Ochola, E. Effect of varying node mobility in the analysis of black hole attack on MANET reactive routing protocols. In Proceedings of the 2016 Information Security for South Africa (ISSA), Johannesburg, South Africa, 17-18 August 2016; pp. 62-68.

8. Felipe, L.; Aguiar, U.; Tripp, C.; Barcelona, B. Design and Implementation of Routing Protocols with Anonymity for Vehicular Ad-Hoc Networks in Urban Environments. Master's Thesis, Universitat Politècnica de Catalunya, Barcelona, Spain, 2012.

9. Istikmal. Analysis and Evaluation Optimization Dynamic Source Routing (DSR) Protocol in Mobile Adhoc Network Based on Ant Algorithm. In Proceedings of the 2013 International Conference of Information and Communication Technology (ICoICT), Bandung, Indonesia, 20-22 March 2013; pp. 400-404.

10. Navarro, S. Redes Wireless Ad-Hoc; Capítulo 3; Universidad de Sevilla: Andalucia, Spain, 2006.

11. Sharma, A.; Kumar, R. Performance comparison and detailed study of AODV, DSDV, DSR, TORA and OLSR routing protocols in ad hoc networks. In Proceedings of the 2016 Fourth International Conference on Parallel, Distributed and Grid Computing (PDGC), Waknaghat, India, 22-24 December 2016.

12. Ferreiro-Lage, J.A.; Pereiro, C.; Rubiños, O.; Aguado, F. Analysis of Unicast Routing Protocols for VANETs. In Proceedings of the 2009 Fifth International Conference on Networking and Services, 20-25 April 2009; pp. 518-521.

13. Zhu, J.; Bensaou, B. Power Control Protocols for Wireless. In Algorithms and Protocols for Wireless and Mobile Ad Hoc Networks; John Wiley and Sons, Inc.: Hoboken, NI, USA, 2009; pp. 315-352.

14. ElBatt, T.; Ephremides, A. Joint scheduling and power control for wireless ad-hoc networks. IEEE Trans. Wirel. Commun. 2004, 3, 74-85.

15. Kawadia, V.; Kumar, P.R. Principles and protocols for power control in wireless ad hoc networks. IEEE J. Sel. Areas Commun. 2005, 23, 76-88. 
16. Campolo, C.; Scopigno, R.; Molinaro, A. Vehicular ad hoc Networks; Springer International Publishing: New York, NY, USA, 2015.

17. MediaWiki. NS-3 PHY Layer Models. Available online: https://www.nsnam.org/docs/models/html/wifi.html (accessed on 14 November 2019).

(C) 2019 by the authors. Licensee MDPI, Basel, Switzerland. This article is an open access article distributed under the terms and conditions of the Creative Commons Attribution (CC BY) license (http:/ / creativecommons.org/licenses/by/4.0/). 